\title{
Generation Time Statistics of Escherichia coli B Measured by Synchronous Culture Techniques
}

\author{
By L. D. PLANK ${ }^{1}$ AND J. D. HARVEY' \\ 1 Department of Physics, University of Waikato, Hamilton, New Zealand \\ 2 Department of Physics, University of Auckland, Auckland, New Zealand
}

(Received 12 March 1979)

\begin{abstract}
Synchronous cultures of Escherichia coli B were produced under various environmental conditions. Analysis of the cell number data permitted the characterization of the generation time distribution for these organisms and the estimation of the mother-daughter generation time correlation coefficients. For all growth conditions, the distribution of generation times was found to be symmetrical with a coefficient of variation of $0 \cdot 22 \pm 0 \cdot 02$. The motherdaughter generation time correlation coefficient was significantly negative at doubling times between 40 and $64 \mathrm{~min}$. However, the results for a culture growing in succinate medium at $37^{\circ} \mathrm{C}$, which had a significantly greater generation time, yielded a correlation coefficient close to zero. Within the range of temperatures studied $\left(26\right.$ to $\left.37^{\circ} \mathrm{C}\right)$, no significant effect on the correlation coefficient was observed.
\end{abstract}

\section{INTRODUCTION}

Considerable variability exists in the generation times of individual bacteria in a population. The nature of the distribution of these generation times has been the subject of a number of studies (Kelly \& Rahn, 1932; Powell, 1955, 1956, 1958; Powell \& Errington, 1963; Kubitschek, 1962, 1971; Schaechter et al., 1962). These workers observed the individual organisms directly and for Escherichia coli, for example, unequivocal conclusions as to the form of the generation time distribution could not be drawn. Considerable uncertainty also exists concerning the nature of the correlations between the generation times of related organisms, particularly those between mother and daughter cells (Powell, 1955, 1956; Powell \& Errington, 1963; Kubitschek, 1962, 1966; Schaechter et al., 1962).

The resolution of the uncertainty surrounding the form of the generation time distribution and the parent-progeny correlations in generation time for $E$. coli is the subject of this paper. Our approach follows that of Harvey $(1972 a, b)$ through which the analysis of synchronous culture growth data permits the extraction of the generation time distribution and the correlations between the generation times of parent and progeny cells. The attraction of the method lies in the accuracy with which these statistical parameters may be obtained and in the comparative ease with which the dependence of these parameters on growth conditions may be studied. The latter effects would be prohibitively tedious to investigate by direct observation.

Synchronous cultures were produced by a modification of the density gradient centrifugation technique described by Mitchison \& Vincent (1965). This technique gave reproducible results and excellent synchrony with a wide variety of growth conditions and media. 


\section{METHODS}

Bacteria and growth conditions. Escherichia coli strain B (from the collection of the Department of Cell Biology, University of Auckland) was maintained on slopes of Difco nutrient agar. All cultures were grown in the modified M9 salts medium of Gudas \& Pardee (1974), with glucose $(0.4 \%, \mathrm{w} / \mathrm{v})$, sucrose $(0.5 \%, \mathrm{w} / \mathrm{v})$, glycerol $(0.5 \%, \mathrm{w} / \mathrm{v})$ or sodium succinate $(0.4 \%$, w/v) added as carbon source. Glucose minimal medium was supplemented with methionine and histidine (both at $50 \mu \mathrm{g} \mathrm{ml}^{-1}$ ) to provide faster growing cultures. Cultures were grown aerobically in an orbital incubator (Gallenkamp, London) in Erlenmeyer flasks with a volume at least five times that of the culture. The growth temperature was $37^{\circ} \mathrm{C}$ for the various carbon sources. The temperature could be maintained within $\pm 0 \cdot 2{ }^{\circ} \mathrm{C}$. Cultures in glucose medium were also grown at temperatures in the range 26 to $37^{\circ} \mathrm{C}$ to obtain different growth rates. In our cultures, bacterial division

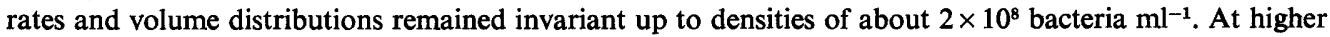
densities, the mean cell volume decreased with time. Cell volume distributions were recorded at regular intervals during the growth of a synchronously dividing culture (Plank, 1978).

Preparation of synchronous cultures. An overnight culture was diluted into fresh medium $(100 \mathrm{ml})$ containing the appropriate carbon source and grown for at least eight generations to a final concentration of between $5 \times 10^{7}$ and $2 \times 10^{8}$ bacteria $\mathrm{ml}^{-1}$. The method of Mitchison \& Vincent (1965) was used to select synchronous sub-populations from the parental cultures. The parent culture was centrifuged at $7000 \mathrm{~g}$ for $5 \mathrm{~min}$ and the resulting pellet was resuspended in 0.5 to $1.5 \mathrm{ml}$ growth medium and then layered on a $40 \mathrm{ml}$ linear density gradient (Britten \& Roberts, 1960) in a $23 \times 110 \mathrm{~mm}$ glass centrifuge tube. [Gradients of sucrose, glucose, glycerol and Ficoll (Type 70; Sigma) were used depending on the carbon source in the growth medium.] Gradients were centrifuged for 6 to $18 \mathrm{~min}$ in a swinging bucket centrifuge (Super Minor; MSE, Crawley, Sussex) at 1500 to $2200 \mathrm{~g}$, generally at room temperature. The duration and speed of centrifugation depended on the cell size, temperature and gradient material and was chosen to give a turbid band extending from about $3 \mathrm{~cm}$ below the meniscus to a depth of about $6 \mathrm{~cm}$. A sample of the smallest organisms $(0.5$ to $1 \mathrm{ml}$, about 1 to $3 \%$ of those loaded) was removed from the upper region of the visible band and was inoculated into prewarmed conditioned growth medium $(15$ to $20 \mathrm{ml})$ to give $0.4 \times 10^{7}$ to $1 \times 10^{7}$ bacteria $\mathrm{ml}^{-1}$. The conditioned medium was parent growth medium filtered to remove residual organisms.

To obtain asynchronous control cultures, bacteria remaining in the band were mixed and a small sample was inoculated into parent growth medium.

Bacterial counts and volumes. The numbers of bacteria were counted with a Coulter model $Z_{\mathrm{BI}}$ particle counter fitted with a commercial $30 \mu \mathrm{m}$ orifice (Coulter Electronics, Harpenden, Herts.). A pulse height analyser constructed in this laboratory (Rackham, 1977) was linked to the counter to provide volume distributions. Culture samples $(0.2 \mathrm{ml})$ were removed with a micropipette and diluted 50 -fold into $0.1 \mathrm{M}-\mathrm{HCl}$ for counting.

To maintain low background counts, all media and electrolyte solutions were prefiltered through $0.22 \mu \mathrm{m}$ pore-size membrane filters (Millipore). Background counts were estimated for the synchronous and control cultures by performing the dilution and counting operations on the conditioned medium just prior to inoculation. Coincidence corrections become important at the higher count rates and these were applied according to the formula of Princen \& Kwolek (1966). The equation derived by these authors was fitted to experimental data obtained over a wide range of count rates.

Data analysis. The general approach to the analysis of the data using numerical differentiation has been described previously (Harvey, 1972a,b). A numerical differentiation applied to the synchronous culture data will be strongly influenced by the sharp fluctuations in the data points. These are caused solely by experimental errors, since it is expected on physical grounds that in a system containing a large number of cells the concentration should vary smoothly with time. The technique of smoothing by digital filtering, used in this paper, has been described in detail by Harvey $(1972 b)$. The derivatives were calculated using a six-point numerical differentiation formula from the smoothed points representing the bacterial concentrations. The uncertainties attached to the parameter values (Table 1) were found as follows. The analysis of actual experimental data was simulated as closely as possible with noisy data generated analytically and bearing close resemblance to the former. Such data were produced by integration of a sum of Gaussian functions, the parameters of which had similar values to those found for particular experiments. In most cases, four Gaussian functions were summed to provide satisfactory data through the third generation. Each data point generated by the integration routine was multiplied by a random error, these errors being normally distributed with a mean of unity and a standard deviation of 0.015 . The latter value was chosen since estimates of the experimental uncertainty associated with any one point arising as a result of sampling, diluting and counting errors were close to $1.5 \%$. The smoothing, differentiation and non-linear least squares fitting of a sum of Gaussian functions was then carried out in the normal way. Twenty to thirty repetitions of this process of sampling from a random number generator and subsequent analysis yielded measures of the dispersion 
in each of the parameters sought. Details of the non-linear least squares routine are given in Plank (1978).

\section{THEORY}

Harvey $(1972 a)$ has presented a mathematical description of synchronous bacterial growth in which the time derivative of the total number of organisms in a synchronous culture is expressed as the sum of a series of functions related to the generation time distribution of individual organisms. That is,

$$
\frac{\mathrm{d} N(T)}{\mathrm{d} T}=N_{0} \sum_{j=1}^{\infty} F_{j}(T)
$$

where $N(T)$ is the total number of organisms in the culture at time $T$ and $N_{0}$ is the initial number of first generation organisms. In equation (1),

$$
F_{j}(T)=2^{j-1} G_{j}(T)
$$

where $G_{j}(T)$ represents the distribution (normalized) of the times of division of the $j$ th generation organisms with mean division time $\tau_{j}$ and standard deviation $\sigma_{j}$. Provided the initial organisms were collected without bias with respect to parent generation times, $F_{1}(T)$ is just the generation time distribution $f(T)$ of the organisms in the culture having mean and standard deviation $\tau$ and $\sigma$, respectively. In general,

$$
2^{1-j} \int_{0}^{\infty} T F_{j}(T) \mathrm{d} T=j \tau
$$

and

$$
2^{1-j} \int_{0}^{\infty}(T-j \tau)^{2} F_{j}(T) \mathrm{d} T=\sigma_{j}^{2}=j \sigma^{2}+2 \sigma^{2} \sum_{k=1}^{j-1}(j-k) \rho^{(k}
$$

In equation (4), $\rho^{(1)}$ is the mother-daughter generation time correlation coefficient, $\rho^{(2)}$ is the mother-granddaughter generation time correlation coefficient, and so on.

Harvey $(1972 a)$ considered the effects of imperfect synchronization at $T=0$ by imagining that new organisms are introduced over a period of time with a certain distribution having variance $\sigma_{0}{ }^{2}$. When the fraction of smallest organisms selected by the gradient centrifugation procedure exhibits some dispersion in cell ages we may account for this imperfect selection by the addition of $\sigma_{0}{ }^{2}$ to the variances of $F_{j}(T)$. Explicitly,

$$
\begin{aligned}
& \sigma_{1}^{2}=\sigma^{2}+{\sigma_{0}}^{2} \\
& {\sigma_{2}}^{2}=2 \sigma^{2}\left[1+\rho^{(1)}\right]+\sigma_{0}^{2} \\
& {\sigma_{3}^{2}}^{2}=3 \sigma^{2}+2 \sigma^{2}\left[2 \rho^{(1)}+\rho^{(2)}\right]+{\sigma_{0}}^{2}
\end{aligned}
$$

\section{RESULTS}

One of five different synchronous culture experiments carried out with $E$. coli $\mathrm{B}$ in glucose/ salts medium at $37^{\circ} \mathrm{C}$ is illustrated in Fig. $1(a)$. The asynchronous control and the synchronous culture showed the same parallel trends in average growth rate. Since this strain of $E$. coli can utilize sucrose, gradients for glycerol- and succinate-grown bacteria were prepared from glycerol and Ficoll, respectively, to avoid a nutritional shift-up. Representative results for various carbon sources are illustrated in Fig. $1(b, c, d)$. With glucose as the carbon source, synchronous cultures obtained at various temperatures between 26 and $37^{\circ} \mathrm{C}$ had different doubling times; a typical example is shown in Fig. 1(e).

\section{Data analysis}

Smoothing and differentiating the experimental concentration curve of experiment $\mathrm{A}$ (Fig. $2 a$ ) yielded the results shown in Fig. $2(b)$ in which digital filter cut-off wavelengths of seven, eight and nine data point spacings have been used. Over this range of cut-off wavelengths, there was little change in the derivative curves. Figure $2(b)$ illustrates the typical form assumed by the functions $F_{j}(T)$ defined by equation (1). It is apparent from the figure that there is considerable interference between the tails of the functions $F_{j}(T)$. Thus, for obtaining estimates of the moments of the distributions, the straightforward summation procedure used by Harvey $(1972 b)$ will be subject to considerable errors. An alternative 


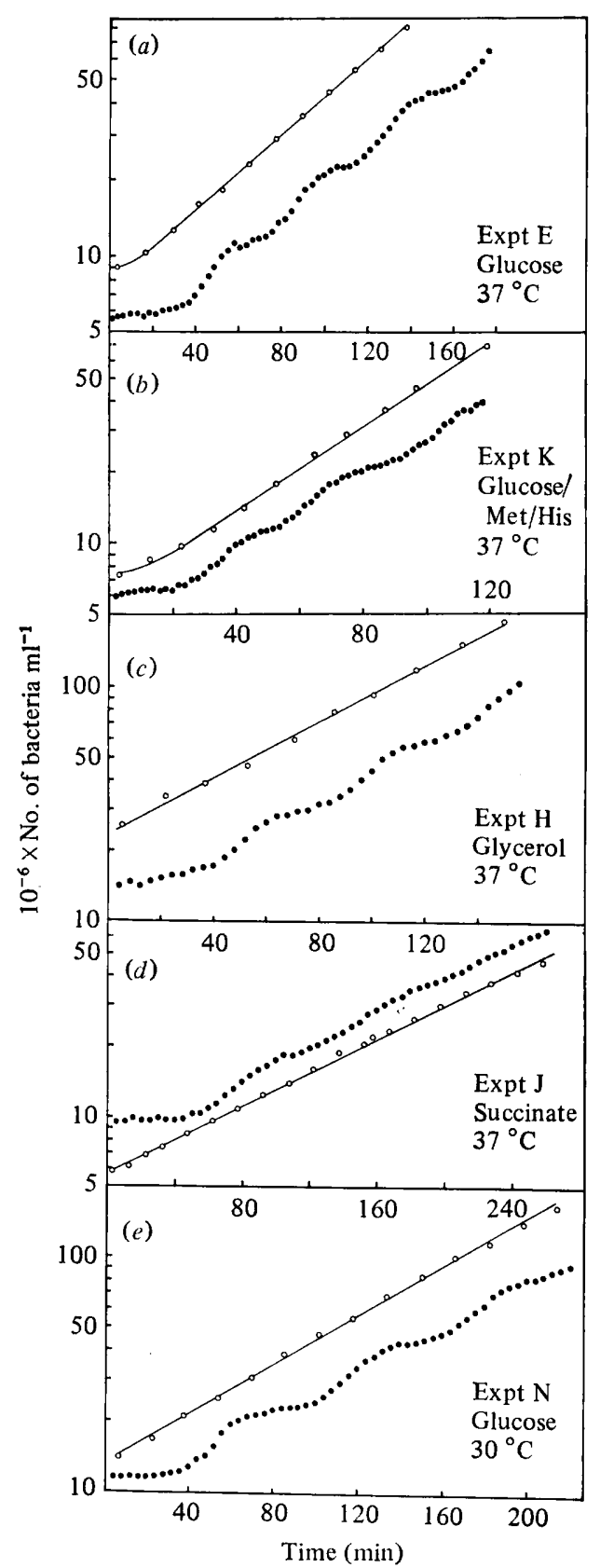

Fig. 1. Logarithmic plots of cell concentration in synchronous cultures (O) and asynchronous control cultures $(O)$ of $E$. coli $\mathrm{B}$. The zero on the time scale corresponds to the time of inoculation of the sample from the gradient into the prewarmed conditioned medium. (a) Experiment $\mathrm{E}$ (see Table 1): glucose-grown bacteria at a density of $10^{8} \mathrm{ml}^{-1}$ were concentrated and banded $(2100 \mathrm{~g}$ for $9 \mathrm{~min}$ ) in a 5 to $30 \%(\mathrm{w} / \mathrm{v})$ sucrose gradient (prepared in growth medium) at $37^{\circ} \mathrm{C}$ and $1 \%$ of the cells loaded were recovered to yield the synchronous culture. (b) Experiment $\mathrm{K}$ : bacteria grown in glucose/salts medium supplemented with methionine and histidine were banded in a sucrose gradient at $2100 \mathrm{~g}$ for $6 \mathrm{~min}$. (c) Experiment $\mathrm{H}$ : glycerol-grown bacteria were removed from a 10 to $50 \%$ $(\mathrm{w} / \mathrm{v})$ glycerol gradient after banding at $1500 \mathrm{~g}$ for $15 \mathrm{~min}$. (d) Experiment $\mathrm{J}$ : succinate-grown bacteria were banded in a 5 to $15 \%(\mathrm{w} / \mathrm{v})$ Ficoll gradient at $2100 \mathrm{~g}$ for $10 \mathrm{~min}$. (e) Experiment $\mathrm{N}$ : bacteria grown at $30^{\circ} \mathrm{C}$ in glucose/salts medium were banded in a sucrose gradient at $1500 \mathrm{~g}$ for $15 \mathrm{~min}$. 


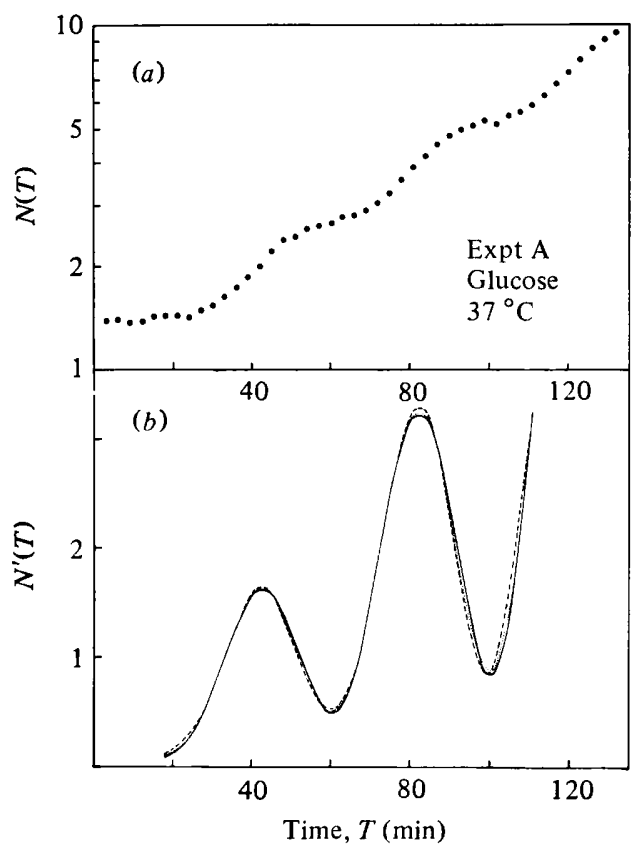

Fig. 2. (a) Logarithmic plot of the bacterial concentration in a synchronous culture established by banding glucose-grown bacteria in a 5 to $30 \%(\mathrm{w} / \mathrm{v})$ sucrose gradient at room temperature at $1500 \mathrm{~g}$ for $12 \mathrm{~min}$ (experiment A, see Table 1). (b) Derivative of the bacterial concentration shown in (a) extracted by digital filtering with cut-off wavelengths of seven $(---)$, eight $(\cdots)$, and nine $(-)$ data point spacings. The ordinate scales are in arbitrary units.

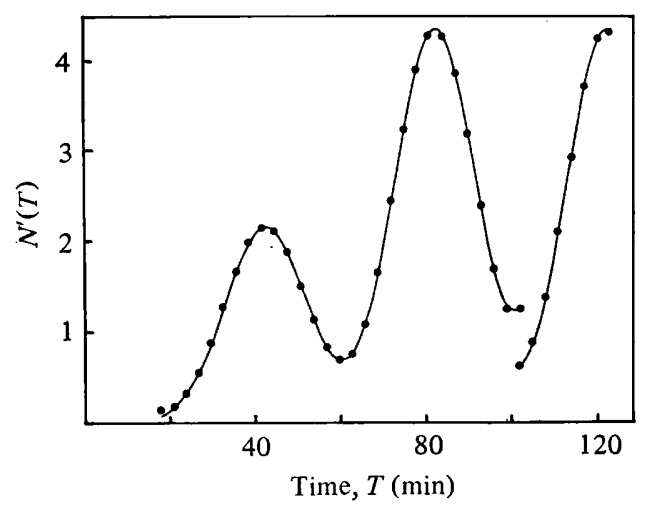

Fig. 3. Sum of three Gaussian functions (- - fitted to the data of Fig. 2(b) for a digital filtering cut-off wavelength of eight data point spacings. The ordinates are in arbitrary units and have been divided by two for times greater than $102 \mathrm{~min}$.

method was therefore desirable. Examination of Fig. 2(b) reveals the lack of any marked skewness in $F_{1}(T)$ or $F_{2}(T)$ and the Gaussian nature of these peaks immediately suggests fitting a sum of normal frequency functions to the curve. Although the non-truncated Gaussian distribution is defined over the whole real line, we can justify its use here because of the negligible values taken at negative times. The means and variances of the distributions $F_{j}(T)$ will then be given directly by the best fit of a sum of Gaussian functions to the derivative curve.

Figure 3 illustrates the result of fitting a sum of three Gaussian functions to the data 
represented in Fig. 2(b) for a digital filtering cut-off wavelength of eight data point spacings. The fit is clearly satisfactory and shows directly the absence of any significant skewness in $F_{1}(T)$ or $F_{2}(T)$. The parameters of the functions $F_{j}(T)$ obtained in this way for all experiments are listed in Table 1, together with the computed values of the coefficient of variation of the generation time distribution and the mother-daughter generation time correlation coefficient (assuming $\sigma_{0}^{2}$ is zero).

The glucose-grown cultures of experiments $\mathrm{D}$ and $\mathrm{E}$ were extended beyond four generations of growth to allow the estimation of $\rho^{(2)}$, the mother-granddaughter generation time correlation coefficient. The fitting procedure extended to the sum of four Gaussian functions together with the error simulation method yielded the estimates (neglecting $\sigma_{0}^{2}$ ) $0 \cdot 03 \pm 0 \cdot 24$ (D) and $-0.02 \pm 0.23(\mathrm{E})$. The precision in the data is thus insufficient to enable this parameter to be estimated reliably. The values quoted are consistent with the hypothesis that $\rho^{(2)}=$ $\left[\rho^{(1)}\right]^{2}$, that is, $\rho^{(2)}$ arises solely from the mutual correlation of the mother and granddaughter cell generation times with the daughter cell generation time.

Comparing the values of $\tau_{1}$ and the difference $\tau_{2}-\tau_{1}$ (Table 1), it is clear that, of the glucose-grown cultures at $37^{\circ} \mathrm{C}$ (experiments A to E), three exhibited a lengthening of the first generation and this was not related to the absence of growth medium in the gradients. The performance of all operations at $37^{\circ} \mathrm{C}$ did not eliminate this lag, which also appeared in the control culture of experiment $\mathrm{E}$. This phenomenon is commonly observed when bacteria are exposed to sucrose (Burdett \& Murray, 1974). In contrast, in sucrose minimal medium (experiments $F$ and $G$ ), a marked shortening of the first generation occurred presumably due to continued growth of the bacteria during centrifugation in the gradient. The asynchronous control cultures for succinate medium (experiments I and J) exhibited doubling times in the range 80 to $83 \mathrm{~min}$. The significantly shorter interval for the second generation growth of experiment I suggests some perturbation of the growth pattern of these bacteria. Owing to the considerable overlap of the functions $F_{2}$ and $F_{3}$ in these experiments, some difficulty was experienced in estimating $\sigma_{3}$ (and $\sigma_{2}$ in the case of experiment $\mathrm{J}$ ) by the curve fitting procedure. The smoothing and differentiation operations were very sensitive to small fluctuations in the data points in the overlap region. A similar situation existed in the analysis of the low temperature culture (experiment $\mathrm{O}$ ). The variance of $F_{3}$ was not well defined in experiments $\mathrm{L}$ and $\mathrm{M}$ because the data had been extended only a few points into $F_{3}$.

\section{DISCUSSION}

The results presented here strongly support a distribution of generation times of normal form. Over the range of growth rates studied (doubling times from 30 to $90 \mathrm{~min}$ ) with growth temperatures between 26 and $37^{\circ} \mathrm{C}$, the normal curve provides a satisfactory fit to the generation time distribution data. For all growth rates and ambient conditions, the coefficient of variation of the generation time distribution for $E$. coli $\mathrm{B}$ is constant, within experimental error, and estimated to be $0.22 \pm 0.02$. With the exception of the extremes of slow growth (doubling times of 80 to $90 \mathrm{~min}$ ) and rapid growth (doubling time of $30 \mathrm{~min}$ ), the mother-daughter generation time correlation coefficient is essentially constant and significantly negative at all the other growth rates studied. The weighted mean value of this correlation coefficient for the glucose-grown cultures at $37^{\circ} \mathrm{C}$ (doubling times close to $40 \mathrm{~min}$ ) is $-0 \cdot 47 \pm 0.06$. In contrast, a marked change occurs in the patterns of growth of the synchronous cultures in which succinate is the carbon source. In experiment $\mathrm{J}$, synchrony rapidly weakened after the first generation of growth, and it was not possible to estimate the mother-daughter generation time correlation coefficient using the curve fitting procedure. The rapid loss of synchrony in this culture suggests that $\rho^{(1)}$ has a value close to zero and corroboration of this is provided in Fig. 4(a). The continuous curve represents a culture in which no dependence exists between the generation times of mother cells and their progeny. The dashed curve, on the other hand, pertains to a culture having the same underlying 


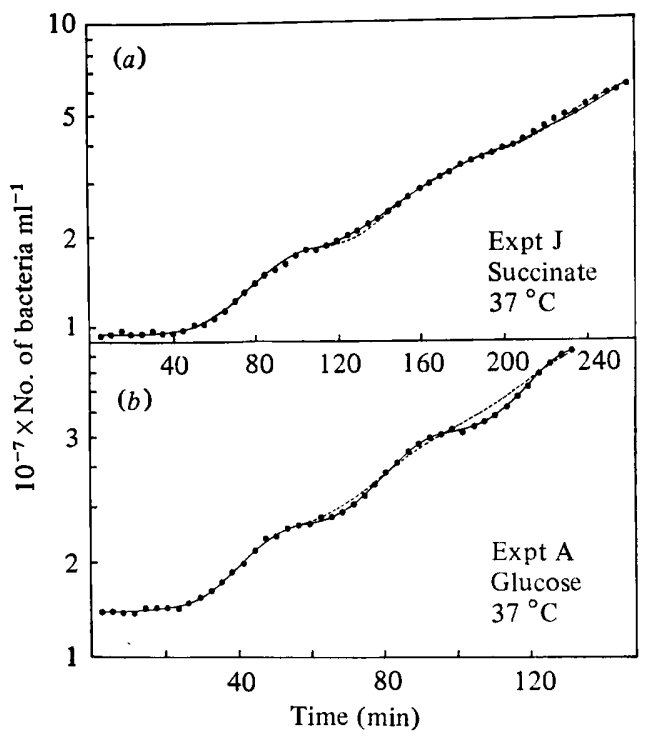

Fig. 4. (a) Experimental results for a succinate-grown synchronous culture (experiment $\mathrm{J}$, see Table 1 ; ) and idealized concentration curves for mother-daughter generation time correlation coefficients having the values zero ( - and $-0.4(--)$. (b) Experimental results for a glucosegrown synchronous culture (experiment A, see Table $1 ; \boldsymbol{O})$ and concentration curves derived for values of the mother-daughter generation time correlation coefficient of zero $(---)$ and -0.5 (一).

generation time distribution, in which the correlation coefficient between the generation times of mother and daughter cells has the value $-0 \cdot 4$. During the growth of the second generation cells, the continuous curve evidently follows the data more closely and a value for $\rho^{(1)}$ close to zero is a reasonable assumption. A similar comparison is shown in Fig. 4(b) for the glucose-grown synchronous culture of experiment A for which the curve fitting procedure was used successfully. These curves show clearly the magnitude of the effects involved and illustrate the need for extreme precision in the estimation of cell concentrations. In regard to the mother-granddaughter generation time correlation coefficient, it is difficult to see, using present counting techniques, how improved precision can be attained in the estimates reported here. As such, the nature of correlations extending over more than two generations must remain inaccessible using synchronous culture techniques. The difficulty in estimating $\rho^{(2)}$ arises because it involves the difference between two nearly equal terms, each having an associated uncertainty of, generally, at least $10 \%$.

The analysis by Harvey (1972 b) of some results of Helmstetter (1969) also indicated a symmetrical generation time distribution for $E$. coli $\mathrm{B} / \mathrm{r}$ with a mother-daughter generation time correlation coefficient of $-0 \cdot 40$. For this strain the coefficient of variation for the generation time distribution was $0 \cdot 17$. Helmstetter (1969) used the membrane elution procedure (Helmstetter \& Cummings, 1964) to provide a synchronous culture. Shehata \& Marr (1970) also used this technique to produce synchronous cultures of three different strains of $E$. coli growing in glucose minimal medium at $30^{\circ} \mathrm{C}$. These workers fitted curves to the cell number data by inspection and then extracted generation time distributions with coefficients of variation in the range $0 \cdot 18$ to 0.22 and which were not appreciably skewed.

Other workers have employed direct observation to investigate the shape and parameters of the generation time distribution. These experiments have generally indicated a marked positive skewness in the distribution of generation times and statistically significant values for $\rho^{(1)}$ have been difficult to obtain. As has already been pointed out (Harvey, 1972 $b$ ), the 
difficulty in maintaining a uniform environment around cells during microscopic observation very likely leads to discrepancies between these results and those reported in this paper.

We wish to thank Drs D. F. Walls, H. W. Morgan and R. A. Sherlock for helpful discussions. L. D.P. was the holder of an NZUGC Postgraduate Scholarship during the course of this research.

\section{REFERENCES}

Britten, R. J. \& Roberts, R. B. (1960). High resolution density gradient sedimentation analysis. Science 131, 32-33.

Burdett, I. D. J. \& Murray, R. G. E. (1974). Electron microscope study of septum formation in Escherichia coli strains B and B/r during synchronous growth. Journal of Bacteriology 119, 1039-1056.

Gudas, L. J. \& Pardee, A. B. (1974). Deoxyribonucleic acid synthesis during the division cycle of Escherichia coli: a comparison of strains $\mathrm{B} / \mathrm{r}$, $\mathrm{K}-12,15$, and $15 \mathrm{~T}^{-}$under conditions of slow growth. Journal of Bacteriology 117, 1216-1223.

HARVEY, J. D. (1972a). Synchronous growth of cells and the generation time distribution. Journal of General Microbiology 70, 99-108.

HARVEY, J. D. (1972 b). Parameters of the generation time distribution of Escherichia coli B/r. Journal of General Microbiology 70, 109-114.

HelmstetTER, C. E. (1969). Methods for studying the microbial division cycle. Methods in Microbiology 1, 327-363.

Helmstetter, C. E. \& Cummings, D. F. (1964). An improved method for the selection of bacterial cells at division. Biochimica et biophysica acta 82, 608-610.

KELLY, C. D. \& RAHN, O. (1932). The growth rate of individual bacterial cells. Journal of Bacteriology 23, 147-153.

KUBITSCHEK, H. E. (1962). Normal distribution of cell generation rate. Experimental Cell Research 26, 439-450.

KUBITSCHEK, H. E. (1966). Generation times: ancestral dependence and dependence upon cell size. Experimental Cell Research 43, 30-38.

KuBITSCHEK, H. E. (1971). The distribution of cell generation times. Cell Tissue Kinetics 4, 113-122.
Mitchison, J. M. \& Vincent, W. S. (1965). Preparation of synchronous cultures by sedimentation. Nature, London 205, 987-989.

Plank, L. D. (1978). Generation time statistics of Escherichia coli using synchronous culture techniques. D.Phil. thesis, University of Waikato, New Zealand.

Powell, E. O. (1955). Some features of the generation times of individual bacteria. Biometrika 42, $16-44$.

Powell, E. O. (1956). Growth rate and generation time of bacteria, with special reference to continuous culture. Journal of General Microbiology 15, 492-511.

Powell, E. O. (1958). An outline of the pattern of bacterial generation times. Journal of General Microbiology 18, 382-417.

Powell, E. O. \& Errington, F. P. (1963). Generation times of individual bacteria: some corroborative measurements. Journal of General Microbiology 31, 315-327.

Princen, L. H. \& Kwolek, W. F. (1966). Coincidence corrections for particle size determinations with the Coulter counter. Review of Scientific Instruments 36, 646-653.

RACKHAM, S. J. (1977). A pulse height analyser for displaying Coulter counter particle size distributions. M.Sc. thesis, University of Waikato, New Zealand.

Schaechter, M., Williamson, J. P., Hood, J. R., JR \& Koch, A. L. (1962). Growth, cell and nuclear divisions in some bacteria. Journal of General Microbiology 29, 421-434.

Shehata, T. E. \& MARR, A. G. (1970). Synchronous growth of enteric bacteria. Journal of Bacteriology 103, 789-792. 